in pre-therapy lesions from patients who achieved pCR compared to the pre-therapy lesions from patients who did not achieve $\mathrm{pCR}^{6}$. It may be that, like in melanoma, the previously primed $\mathrm{CD}^{+} \mathrm{T}$ cells become exhausted and then, upon PD-1 blockade, are rapidly reinvigorated resulting in immune response. Further understanding of the subtypes of $\mathrm{CD}^{+} \mathrm{T}$ cells in bladder cancer would clarify the mechanism.

The findings here warrant validation in an independent dataset. Biomarkers such as TMB, DDR alterations, PD-L1 and molecular subtypes, previously reported to enrich for immune response to atezolizumab in metastatic disease, did not do so in the neoadjuvant setting in this study ${ }^{5}$. Although the association between the cytotoxic $\mathrm{T}$ cell signature and pCR was significant, a larger trial size would further confirm this. Development of consistent scoring methods to determine 'high' versus 'low' CD8 expression and standard $\mathrm{T}$ cell signatures will become important to translate this finding into clinical use. Although some of the immune biomarkers conflict with the PURE-01 data, where PD-L1-positive and high-DDR tumors had a greater response to immunotherapy, the biomarker data were reported in only 50 patients in PURE-01, and to date neither set of findings have been independently validated in a subsequent cohort. Additionally, the use of different immunotherapy drugs, differences in patient populations and three instead of two cycles of neoadjuvant immunotherapy may contribute to some differences between the two studies.

As neoadjuvant immunotherapy and combination chemoimmunotherapy regimens demonstrate encouraging outcomes in UC, identifying validated biomarkers to predict immune response will inform treatment decisions. The work by Powles et al. ${ }^{5}$ is a step in that direction. Ultimately being able to determine whom to offer neoadjuvant immunotherapy versus chemotherapy versus combination therapy would allow us to maximize benefit, minimize risk and improve patient outcomes.

Pooja Ghatalia and Elizabeth Plimack (D)* Fox Chase Cancer Center, Philadelphia, PA, USA. *e-mail: elizabeth.plimack@fccc.edu

Published online: 4 November 2019

https://doi.org/10.1038/s41591-019-0645-6

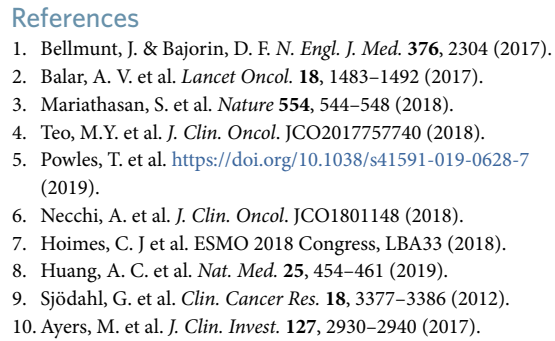

\title{
MICROBIOME
}

\section{The differing roles of lactobacilli in critical illness}

\author{
New findings regarding the risks of over-the-counter probiotics in critically ill patients underscore the need for \\ microbiota-targeted therapies that are based on efficacy in a disease- and host-specific context.
}

\section{Bastiaan W. Haak and W. Joost Wiersinga}

T here has been a surge in the worldwide use of over-the-counter probiotics in recent years in tandem with an increased understanding of the vital role of the gut microbiota on human health ${ }^{1}$. The commercial development of these formulations has progressed into multibillion-dollar endeavors, and the potential benefits of probiotics have been studied in a wide range of disorders. In the intensive care unit (ICU), studies using probiotics have specifically focused on their administration for the prevention of ventilator-associated pneumonia, pancreatitis and sepsis, as well as the treatment of neonatal late-onset sepsis $(\mathrm{LOS})^{2-4}$. However, differences in the size and methodological rigor of the individual studies conducted, as well as large variations in the type, duration and dose of administered probiotic strains, continue to limit the strength of any conclusions regarding the effects of these formulations ${ }^{1}$.

Although that probiotics are generally considered safe, concerns have been raised that the administration of these live organisms could contribute to antibiotic resistance through horizontal transfer of antibiotic-resistance genes in the gastrointestinal environment ${ }^{5}$. In addition, administration of probiotic formulations has been associated with an increased risk of bacteremia in preterm neonates and ICU patients ${ }^{6}$. However, it has been challenging to determine a causal link between administration of probiotics with bacteremia, as these species-predominantly Lactobacillus spp. and Bifidobacterium spp.-are also common constituents of the human residential gut flora, which could therefore have been the underlying source of bacteremia, rather than probiotic administration itself. In this issue, two contributions shed further light on the contrasting roles of lactobacilli. While one study shows that Lactobacillus rhamnosus GG strains can indeed directly cause bacteremia and adaptively evolve in critically ill patients ${ }^{7}$, a second shows that the development of late-onset sepsis in a mouse model through intestinal overgrowth of Klebsiella pneumoniae can be prevented by administration of certain other Lactobacillus strains ${ }^{8}$.

Yelin and colleagues ${ }^{7}$ carried out a large epidemiological study using surveillance data that was collected from over 22,000 ICU patients at Boston Children's Hospital between 2009 and 2014. A total of 522 ICU patients received the probiotic L. rhamnosus GG as part of their treatment (LGG), most commonly because they had been taking it prior to ICU admission. Of note, there were no ICU-specific guidelines for probiotic administration. The authors found that patients receiving this probiotic formulation had significantly higher risk of developing L. rhamnosus bacteremia. The authors then carried out whole-genome sequencing of the bacteria to determine strain-level similarity, and found that the blood isolates of $L$. rhamnosus were phylogenetically inseparable from probiotic isolates administered at that time, confirming that these probiotic strains were indeed the source of bacteremia. Additionally, the authors found some mutations in the blood isolates in which they differed from 

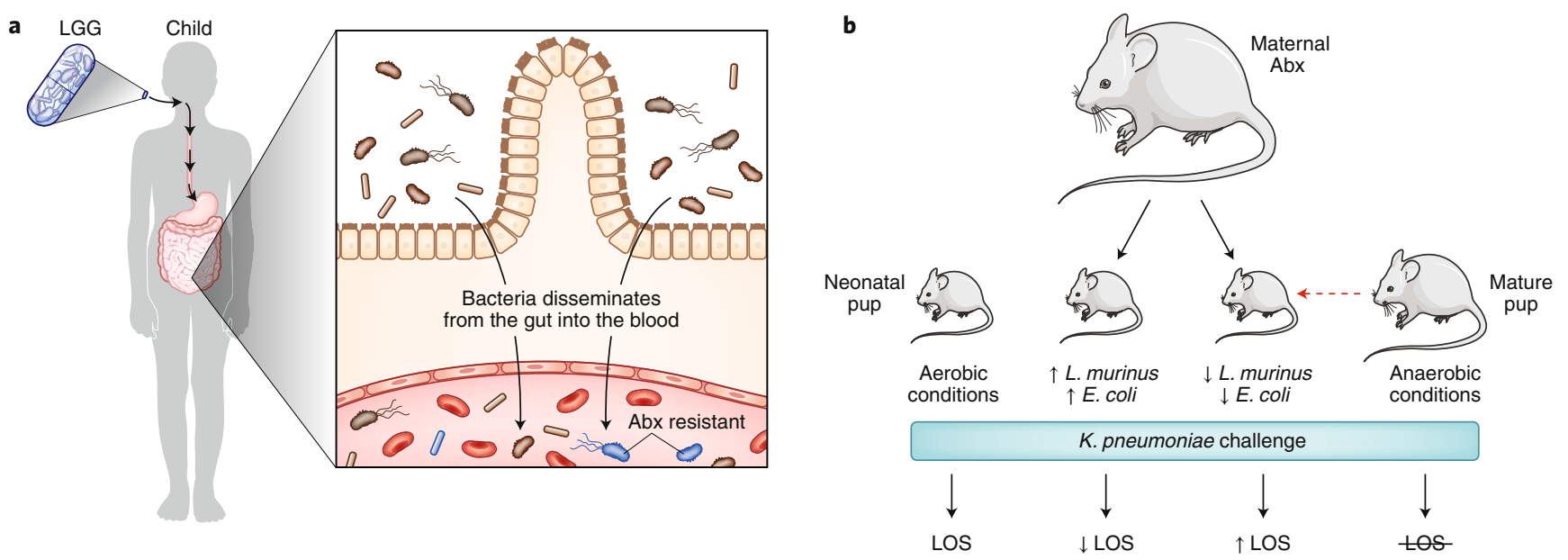

Fig. 1 | Understanding the contrasting roles of probiotics in critical illness. (a) Yelin and colleagues ${ }^{7}$ show in humans that L. rhamnosus GG strains can directly cause bacteremia and adaptively evolve in critically ill patients becoming antibiotic resistant. (b) Singer and colleagues ${ }^{8}$ show that the development of lateonset sepsis due to oxygen-dependent overgrowth of $K$. pneumoniae can be prevented by certain Lactobacillus strains. They found that aerobic conditions in the immature neonatal gut were major drivers of $K$. pneumoniae overgrowth, whereas older pups with more mature microbiome communities-containing anaerobic bacteria-had resistance to the disease. However, administration of mature microbiota to neonatal pups led to limited intestinal engraftment of anaerobic communities. Abx, antibiotics; E. coli, Escherichia coli; K. pneumoniae, Klebsiella pneumoniae; L. murinus, Lactobacillus murinus; LGG, Lactobacillus rhamnosus GG; LOS, late-onset sepsis.

the probiotic bacteria, suggesting that the probiotic strains could have mutated within the host environment. Strikingly, in one instance they identified a de novo mutation conferring resistance to the antibiotic rifampin in an isolate from a patient who received concurrent treatment with LGG and with the rifampicin derivate rifaximin during the 3 months before developing bacteremia, indicating that this probiotic strain may be susceptible to environmental evolutionary pressure.

Lactobacillus bacteremia occurs infrequently in critical illness, and its clinical significance is poorly defined ${ }^{9}$. However, although Lactobacillus bacteremia was diagnosed in only 6 of 522 ICU patients (1.1\%) receiving LGG-containing probiotics, only 2 of 21,652 ICU patients $(0.009 \%)$ not receiving the LGG probiotic developed Lactobacillus bacteremia, indicating that the risk of bacteremia increased significantly upon probiotic administration. In addition, several key questions remain to be answered, most importantly which underlying mechanisms and risks drive progression to bacteremia, as none of these 6 critically ill patients had factors that would predispose them to infection, such as severe immunosuppression or signs of bowel disintegrity. Nonetheless, these findings fuel the need to develop a deeper mechanistic understanding of how live micro-organisms drive health-promoting effects in order to develop a new generation of probiotics that can be employed with less potential for unwanted consequences.
In another study in this issue, Singer and colleagues ${ }^{8}$ investigate LOS, a neonatal infection in premature infants that is thought to be caused by commensal bacteria and fungi, using a model system in mice. They colonized the gut of neonatal mice with bioluminescent and fluorescent strains of Klebsiella pneumonia so that they could be traced. First, the authors showed that gut overgrowth with $K$. pneumoniae preceded the spread of the pathogen into the systemic circulation, subsequently leading to extra-intestinal infection and LOS. Then, using a combination of germ-free animals without a microbiome and mouse models in which dams were given vancomycin and gentamycin to alter the microbiome inherited by their pups, they found that pups in which Lactobacillus murinus was largely eradicated had a higher susceptibility to $K$. pneumoniae overgrowth and subsequent sepsis, whereas enrichment with L. murinus conferred relative protection. These findings were confirmed by the observation that probiotic administration of L. murinus, and to a lesser extent Lactobacillus johnsoniibut not LGG-provided resistance to $K$. pneumoniae colonization. Of interest, a single probiotic strain of Escherichia coli was similarly effective in preventing dysbiosis, showing that the efficacy of probiotics in preventing infection was not limited to lactobacilli. A later series of experiments suggested that low microbiota diversity and elevated intestinal oxygen levels in the immature neonatal gut were major drivers of K. pneumoniae overgrowth and risk of
LOS, whereas older pups with more mature microbiome communities-containing anaerobic bacteria-displayed resistance to the disease.

These two contributions ${ }^{7,8}$ shed light on the potential therapeutic uses and risks of probiotics. One important takeaway is that host-microbiota interactions remain extremely complex (Fig. 1) and are likely to be specific to both context (e.g., prior antibiotic exposure, intestinal maturation and integrity, age) and disease. Therefore, a one-size-fits-all approach to treating patients with probiotics is not only potentially limited in efficacy, but also likely to be harmful. We foresee that these findings, along with recent discoveries that the commensals Blautia producta and Clostridium scindens are capable of providing colonization resistance against vancomycin-resistant Enterococcus ${ }^{10}$ and Clostridium difficile ${ }^{11}$, respectively, will provide further grounds for recommending that untargeted administration of probiotics should be supplanted by tailored microbiome therapy. This also extends to identifying microbiota-derived secreted factors, cell components and metabolites that could help overcome the challenges of introducing live organisms into the host environment ${ }^{12}$. It is clear that human studies and randomized clinical trials, with an emphasis on therapeutic reproducibility and patient safety, should be highly anticipated as means to translate these exciting preclinical steps into new therapeutic applications. 
Bastiaan W. Haak ${ }^{1}$ and

W. Joost Wiersinga, ${ }^{1,2 *}$

${ }^{1}$ Center for Experimental and Molecular Medicine (CEMM), Amsterdam University Medical CentersLocation AMC, University of Amsterdam, Amsterdam, the Netherlands. ${ }^{2}$ Department of Internal Medicine,

Division of Infectious Diseases, Amsterdam University Medical Centers-Location AMC, University of Amsterdam, Amsterdam, the Netherlands.

*e-mail:W.J.Wiersinga@amsterdamumc.nl
Published online: 7 November 2019

https://doi.org/10.1038/s41591-019-0623-z

References

1. Suez, J., Zmora, N., Segal, E. \& Elinav, E. Nat. Med. 25, 716-729 (2019).

2. Dermyshi, E. et al. Neonatology 112, 9-23 (2017).

3. Panigrahi, P. et al. Nature 548, 407-412 (2017).

4. Manzanares, W., Lemieux, M., Langlois, P. L. \& Wischmeyer, P. E. Crit. Care 20, 262 (2016).

5. Varankovich, N. V., Nickerson, M. T. \& Korber, D. R. Front. Microbiol. 6, 685 (2015).
6. Didari, T., Solki, S., Mozaffari, S., Nikfar, S. \& Abdollahi, M Expert Opin. Drug Saf. 13, 227-239 (2014).

7. Yelin, I. et al. Nat. Med. https://doi.org/10.1038/s41591-019-06269 (2019).

8. Singer, J. R. et al. Nat. Med. https://doi.org/10.1038/s41591-0190640-y (2019).

9. Salminen, M. K. et al. Clin. Infect. Dis. 38, 62-69 (2004).

10. Kim, S. et al. Nature 572, 665-669 (2019).

11. Buffie, C. et al. Nature 517, 205-208 (2015).

12. Depommier, C. et al. Nat. Med. 25, 1096-1103 (2019).

Competing interests

The authors declare no competing financial interests.

\section{SGLT-2 inhibitors in heart failure: a new therapeutic avenue}

Dapagliflozin, a sodium-glucose co-transporter-2 inhibitor, reduced the risk of cardiovascular death or heart failure events in 4,744 patients with chronic heart failure with reduced ejection fraction.

\section{Muthiah Vaduganathan and Javed Butler}

$\mathrm{T}$ he inhibition of sodium-glucose co-transporter-2 (SGLT2), the principal protein responsible for the reclamation of filtered glucose in the proximal renal tubule, has become the target of intense recent pharmacological interest. These therapies were initially designed for glycemic regulation in diabetes mellitus (DM), but their potential scope of application is now recognized to be considerably broader. Phlorizin is a naturally occurring, non-selective SGLT inhibitor that was first extracted in 1835 from the bark of apple trees by French scientists ${ }^{1}$, and it took over 150 years for the development of synthetic, more specific SGLT2 inhibitor analogs. There are now four SGLT2 inhibitors commercially available in the United States. In their initial safety evaluation in patients with type 2 DM as mandated by a US Food and Drug Administration (FDA) regulatory guidance ${ }^{2}$, not only were they all safe with respect to cardiovascular effects, but they also led to unexpected and profound benefits: preventing hospitalization for heart failure (HF) by $25-35 \%{ }^{3}$ and reducing clinically relevant kidney outcomes ${ }^{4}$.

These serendipitous findings $s^{5}$ have spurred swift expansion of clinical trials with SGLT2 inhibitors, which are now being evaluated in patients with heart failure and chronic kidney disease, both with and without DM. SGLT2 inhibitors have become one of the most studied cardiometabolic therapies, with completed or ongoing large-scale randomized clinical trials collectively evaluating over 80,000 participants. Recently, the DAPA-HF (Dapagliflozin and Prevention of Adverse Outcomes in Heart Failure) trial ${ }^{6}$, evaluated the SGLT2 inhibitor, dapagliflozin, in individuals with heart failure with reduced ejection fraction (HFrEF), and it is the first trial to demonstrate clinical benefits of this therapeutic class in patients with HF, including among the $\sim 60 \%$ of participants without DM (Fig. 1).

The SGLT2 transporter reclaims 90-95\% of glucose in the proximal convoluted tubule, and its activity is upregulated in hyperglycemia. The modest improvement in glycemic control ( $\sim 0.5-1 \%$ lowering in glycated hemoglobin) with SGLT2 inhibitors is insufficient to explain the early and pronounced benefits of this class on heart failure prevention seen with initial large-scale trials of at-risk patients with type $2 \mathrm{DM}^{7}$. It is intriguing that SGLT2 receptors are not known to be expressed on myocardial cells, and it is thought that they have indirect effects on the cardiovascular system, possibly through systemic effects. Postulated mechanisms include favorable hemodynamic and natriuretic effects, improvements in myocardial energetics,

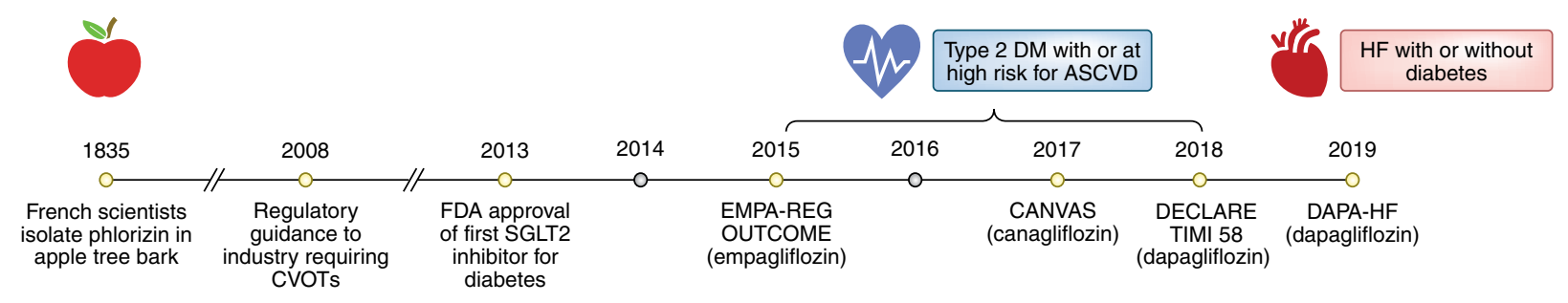

Fig. 1 | A timeline of the discovery and evaluation of SGLT2 inhibitors. ASCVD, atherosclerotic cardiovascular disease; CVOTs, cardiovascular outcomes trials. 